\title{
The Effect of Intermittent Limiting Anodic Current Stimulation on the Electro Activity of Anodic Biofilms
}

\author{
Enren Zhang ${ }^{1^{*}}$, Qingling $\mathrm{Yu}^{1}$, Yongcai Zhang ${ }^{{ }^{*}}$, Keith Scott ${ }^{2}$ and Guowang Diao ${ }^{2}$ \\ ${ }^{1}$ School of Chemistry and Chemical Engineering, Yangzhou University, Yangzhou 225002, China \\ ${ }^{2}$ School of Chemical Engineering and Advanced Materials, Newcastle University, Newcastle NE1 7RU, United Kingdom
}

\begin{abstract}
Electro active biofilms is key components of bio electrochemical systems. Anodic biofilms formed by a potentialadaptive way (i.e., operation with constant external resistance or poised anode potentials) generally lack the ability to adapt to limiting currents. In the present study, a strategy based on intermittent limiting anodic current application was first employed to directly stimulate the current-adaptive growth of anodic biofilms, aiming to obtain electro active biofilms with high current adaptation. Series of intermittent limiting anodic current stimulations exhibit effectiveness for enhancing the electro-activity of anodic biofilms. Porous thick anodic biofilms with less extracellular polymeric substance production could be obtained by series of intermittent limiting anodic currents application from thin biofilms, generating robust anodic biofilms. The high stable power output of $4.35 \mathrm{~W} \mathrm{~m}^{-2}$ could be achieved with robust anodic biofilms at high working current of $9.5 \mathrm{~A} \mathrm{~m}^{-2}$. The present study show that current is a crucial factor influencing the performance of electro-active biofilms, suggesting that an alternative current-adaptive method could be developed for the growth of electro active biofilms with high performance.
\end{abstract}

Keywords: Electro active biofilms; Intermittent current stimulation; Current-adaptive growth; Microbial fuel cells; Bio-electrochemical

\section{Introduction}

Microorganisms capable of achieving extracellular electron transfer, termed exoelectrogens [1], have rapidly attracted increasing attention in bio-electrochemical systems (BESs) for converting wastes into electricity, hydrogen and other valuable chemicals [2-4]. Of particular interest has been systems related to microbial fuel cells (MFCs) [5,6], microbial electrolysis cells [7-9] and on-line microbial monitoring [10-12].

Microbial biofilms developed on the anode play a vital role for sustainable operation of BESs. Anode-attached biofilms accelerate the rate of the complex electrochemical reactions occurring at the interface between the solution and the solid electrode by "catalyzing" the oxidation of waste organic matter and thereby transporting electrons, derived from the oxidation reactions, to the cathode [13]. Unlike chemical catalysts or enzymes employed in other types of electrochemical systems, anode-attached biofilms exhibit both electrochemical catalysis and microbial metabolism. The development of anodic biofilms is directly related to the adaptive growth of microorganisms, rather than to a random stacking of cells on the anode surface. Thus, it is reasonable to believe that multiple factors, including physical, chemical, and electrochemical operating parameters [13-16], can have a significant effect on the development and electrochemical property of anode-attached biofilms. To activate MFC systems, the most commonly employed method is to, after inoculation with a bacterial source, connect a constant external resistance between the anode and the cathode, thus encouraging growth of electro active microorganisms. Alternatively, some studies employed a poised constant anode potential for the growth of electro active microorganisms [17-20], in order to activate the microbial anode and then to explore the effect of electrode potential on the development of electro active biofilms. In circuits with an external resistance or with poised anode potential, biofilm formation is the processes in which electro-active microorganisms (probably also involving non-electro active strains in systems with mixed cultures) grow adaptively in response to either time-dependent anode potentials (e.g., in constant external resistance operations) or to constant anode potentials (e.g., in poised anode potential operations). However, electro-active biofilms developed in such a potentialadaptive way are generally fragile, lacking the ability to adapt to higher currents. For example, in MFC polarization measurements, currents are often observed to sharply decrease in high current range in some MFCs [21-23]. This lack of adaptation to higher currents in bio-anodes would enable MFCs to exhibit unusual electrochemical behaviors or to fail to function in higher current ranges.

To enhance the high current adaptability of anodic biofilms, alternative methods or approaches to biofilm growth should ideally be developed. However, when bio-anodes were subjected to a continuous limiting current, the anode potential would be rapidly polarized to more positive potentials beyond that suitable for microbial growth, enabling the current-adaptive growth failure. Some strategies based on intermittent connection/disconnection operation were recently developed to modulate the anode potential duration [24], external resistance loading [25,26] and MFC voltage [27]. Inspired by these studies, intermittent limiting anodic currents (ILACs) application was designed to stimulate the current-adaptive growth of anodic biofilms for the first time in the present study. Data showed that biofilms can endure the imposition of ILACs, and that a series of ILAC stimulations has a positive effect on the electrochemical behavior of the subsequent anodic biofilms.

*Corresponding authors: Enren Zhang, School of Chemistry and Chemical Engineering, Yangzhou University, Yangzhou 225002, China, Tel: 86018952568061; Fax: 86051487975244; E-mail: erzhang@yzu.edu.cn

Yongcai Zhang, School of Chemistry and Chemical Engineering, Yangzhou University, Yangzhou 225002, China, Tel: 86018952568061; Fax: 86051487975244; E-mail: zhangyc@yzu.edu.cn

Received June 07, 2017; Accepted June 09, 2017; Published June 11, 2017

Citation: Zhang E, Yu Q, Zhang Y, Scott K, Diao G (2017) The Effect of Intermittent Limiting Anodic Current Stimulation on the Electro Activity of Anodic Biofilms. J Adv Chem Eng 7: 174. doi: 10.4172/2090-4568.1000174

Copyright: @ 2017 Zhang E, et al. This is an open-access article distributed under the terms of the Creative Commons Attribution License, which permits unrestricted use, distribution, and reproduction in any medium, provided the original author and source are credited. 


\section{Materials and Methods}

\section{MFC system}

To avoid possible differences in chemical/microbial conditions for the development of anodic biofilms in individual MFCs, experiments used a multiple anode configuration in the anodic chamber of a single plexi-glass MFC. The MFC consisted of two chambers, each of volume $3 \mathrm{dm}^{3}$ (L) $(10 \mathrm{~cm} \times 10 \mathrm{~cm} \times 30 \mathrm{~cm})$. The two electrode chambers were separated by a cationic exchange membrane (YS-1, Shenzhen) of $160 \mathrm{~cm}^{2}$ area $(8 \mathrm{~cm} \times 20 \mathrm{~cm})$. Both anode and cathode were made of carbon felt of thickness $0.25 \mathrm{~mm}$ (H2315, Freudenberg FCCT KG, Germany). In experiments, duplicate carbon felt anodes were separately fixed in the same anode chamber. For each anode, a saturated calomel electrode (SCE) was installed close to the anode surface ( $\sim \mathrm{cm}$ apart) for subsequent potential measurements. If not stated otherwise, each anode area was $4 \mathrm{~cm}^{2}(1 \mathrm{~cm} \times 2 \mathrm{~cm} \times 2)$. The cathode had a size of 5.0 $\mathrm{cm} \times 10.0 \mathrm{~cm}$. In such a configuration, one small anode and one large cathode constituted an MFC circuit, and all anodes shared common chemical/microbial conditions for the development of anodic biofilms (Figure S1).

\section{MFC operation}

To operate the MFC system, $2.7 \mathrm{~L}$ of catholyte containing $50 \mathrm{mM}$ $\mathrm{K}_{3} \mathrm{Fe}(\mathrm{CN})_{6}$ and $100 \mathrm{mM} \mathrm{KH}_{2} \mathrm{PO}_{4}$ (pH adjusted to 7.0 ) was used in the cathode chamber, and $2.0 \mathrm{~L}$ anodic growth medium and $0.7 \mathrm{~L}$ mixed inoculum, taking from a separately operated (long-term running) MFC anode chamber, were added into the anode chamber. The longterm running MFC was initially inoculated with an anaerobic sludge (sampled from a domestic wastewater treatment plant of Tang Wang in Yangzhou city) and was operated using acetate as electron donor over six months. The growth medium was prepared using the following constituents (in grams per liter of deionized water): $\mathrm{NaAc}, 1.6$; $\mathrm{NaHCO}_{3}, 2.5 ; \mathrm{CaCl}_{2} \cdot 2 \mathrm{H}_{2} \mathrm{O}, 0.1 ; \mathrm{KCl}, 0.1 ; \mathrm{NH}_{4} \mathrm{Cl}, 1.5 ; \mathrm{NaH}_{2} \mathrm{PO}_{4} \cdot \mathrm{H}_{2} \mathrm{O}$, $0.6 ; \mathrm{NaCl}, 0.1 ; \mathrm{MgCl}_{2} \cdot 6 \mathrm{H}_{2} \mathrm{O}, 0.1 ; \mathrm{MgSO}_{4} \cdot 7 \mathrm{H}_{2} \mathrm{O}, 0.1 ; \mathrm{MnCl}_{2} \cdot 4 \mathrm{H}_{2} \mathrm{O}, 0.005 ;$ $\mathrm{NaMoO}_{4} \cdot 2 \mathrm{H}_{2} \mathrm{O}, 0.001$; yeast extract 0.05 . After startup of microbial anodes (indicated by the anode potentials), the MFC system was operated in a flow mode by continuously supplying anodic growth medium at a rate of $2 \mathrm{ml} \mathrm{min}{ }^{-1}$, in order to maintain chemo stat conditions in the anode chamber. For constant resistance operation, a constant resistance was connected to each anode and its corresponding cathode. For ILAC stimulations, the anode and its corresponding cathode were connected in series to a $24 \mathrm{~V}$ DC stabilized power supply (UTP3701, UNI-Trend Group Ltd., Guangdong), an adjustable resistor $(0 \sim 100,000 \Omega)$ and a time-cycle relay (DH48S-S, Omron). The constant ILAC magnitude (ILAC-M) was controlled by adjusting the resistor based on the calculation ILAC-M $=\left(V_{24 \mathrm{~V}}+V_{\mathrm{MFC}}\right) / R_{\text {adj }}$, where $V_{24 \mathrm{v}}$ $V_{\mathrm{MFC}}$ and $R_{\text {adj }}$ are the voltage ( $24 \mathrm{~V}$ ) of the stabilized power supply, the working voltage of the MFC and the adjustable resistance, respectively. Because the variation in $V_{\mathrm{MFC}}$ was less than $0.35 \mathrm{~V}$ (much smaller than $\left.\left(V_{24 \mathrm{~V}}+V_{\mathrm{MFC}}\right)\right)$ during ILAC application, the variation in the ILAC-M could be ignored at a fixed adjustable resistance. The on/off time-cycle of the relay was set $1 \mathrm{~s}$ on and $1 \mathrm{~s}$ off to provide ILAC for the microbial anode. (Figure S1).

\section{Measurements}

The voltage outputs for each anode and cathode pair and the anode potential (measured relative to a saturated calomel electrode (SCE) placed in the anode chamber) were continuously measured using a multiple-channel high-impedance voltmeter (Keithley 2700), and data were recorded in every minute. Linear sweep voltammetry (LSV) for each anode was performed using a potentiostat (CHI 660) with the SCE and the corresponding cathode as reference electrode and counter electrode, respectively. During LSV measurements, all anodes were disconnected from the circuits except for the measured anode. Electrode potentials are reported as values relative to the standard hydrogen electrode (SHE).

For scanning electronic microscopy (SEM), carbon felt anodes were removed from the chamber, and biofilms were fixed with $1 \%$ gluteraldehyde overnight, followed by dehydration with a series of graded ethanol $(30,50,70,80,95$, and $100 \%)$. The anode surface was observed using scanning electronic microscopy (HITACHI S-4800 SEM). Chemical oxygen demand (COD) was measured by a fast digestion spectrophotometric method with a COD digester and photometer (Lianhua 5B-3C, China). Medium $\mathrm{pH}$ was measured by a $\mathrm{pH}$ meter (PHS-3C, Leici, Shanghai).

\section{Results and Discussion}

\section{Anode activation at different external resistance}

Initially, different external resistances $(10 \Omega, 50 \Omega, 100 \Omega, 200 \Omega$, $300 \Omega, 400 \Omega, 500 \Omega, 1000 \Omega$ and $2000 \Omega$ ) were employed to activate the microbial anodes under stable chemo stat conditions with $830 \pm 76$ COD and $6.83 \pm 0.15 \mathrm{pH}$ (Figure S2). The external resistance showed a significant impact on the development of microbial anodes in terms of biofilm formation and electro activity. Lower external resistance generated higher stable currents (Figure 1C), consistent to previous studies [28,29]. Anode potential measurements showed that the increase in voltages could be mainly attributed to the development of the microbial anodes in these anode-limiting MFCs (Figure 1B). The electro catalytic behavior of the activated anodes was characterized by slow LSV $\left(1 \mathrm{mV} \mathrm{s}^{-1}\right)$, after voltage stabilization for approximately 100 hours. Figure 1D shows that all activated anodes exhibited catalytic behavior for the bio-electro oxidation of acetate as shown by the appearance of an oxidation current at an onset potential of $-0.25 \mathrm{~V}$ (vs. SHE) similar to other previous reports $[21,28,30]$. Significant differences in currentpotential profiles were observed for anodes activated using different external resistances. Two types of electrochemical behavior can be distinguished according to current-potential profiles (Figure 1D). For anodes activated with a lower external resistance (LER-Anodes. activated with $10 \Omega, 50 \Omega, 100 \Omega, 200 \Omega$ and $300 \Omega$ ), currents increased monotonically with positive potential shifts, to reach limiting currents in the potential range higher than $c a$. $-0.05 \mathrm{~V}$. In contrast, all anodes activated using higher external resistance (HER-Anodes, activated with $400 \Omega, 500 \Omega, 1000 \Omega$ and $2000 \Omega$ ), produced peak currents at potentials between $-0.1 \mathrm{~V}$ and $0 \mathrm{~V}$. However, currents measured on HER-Anodes decreased significantly in the high potential range that produced stable limiting current for the LER-Anodes. Similar behavior was always been observed in our experimental systems, except that the threshold value of external resistance that differentiates the two types of current-potential profiles changed with anodes of different area (Figure S3). Amongst all activated anodes, the highest electro catalytic current, obtained by slow LSV measurements, was produced by one of the HERAnodes, i.e., the anode-400 (Figure 1C), similar to previous studies in which the highest electro activity was obtained with an intermediate external resistance [28].

\section{Sensitivity of HER-anodes}

The maximum current measured by slow LSV represents the highest catalytic capability that an activated anode may achieve under specific operating conditions. Although high catalytic currents were obtained on some HER-Anodes (e.g., anode-400, anode-500 and anode-1000 in Figure 1D), further experiments demonstrated that HER-Anodes did 

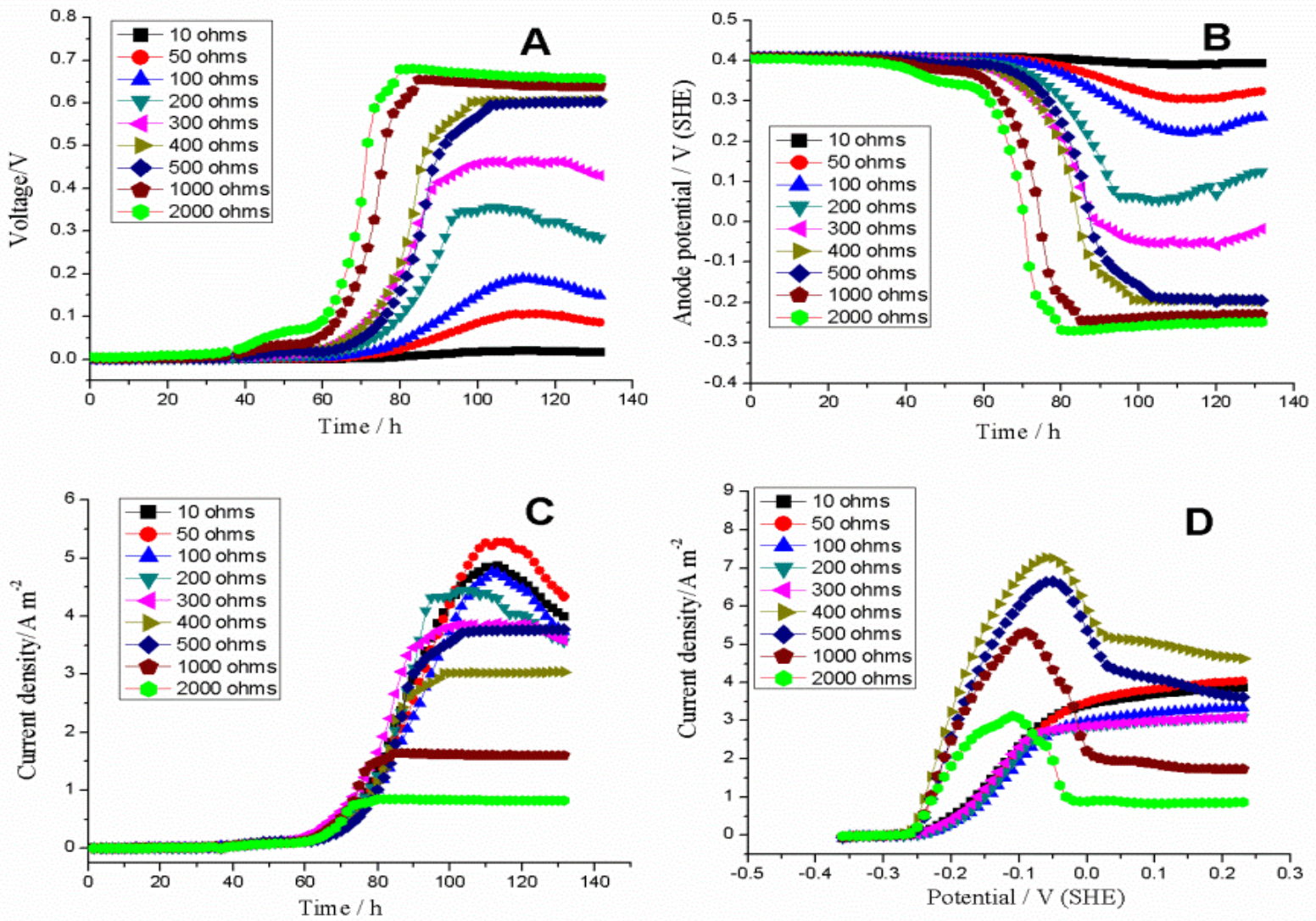

Figure 1: Variations in voltage (A), anode potential (B) and current density (C) for anodes activated with different external resistance (10 $\Omega, 50 \Omega, 100 \Omega, 200 \Omega$, $300 \Omega, 400 \Omega, 500 \Omega, 1000 \Omega$ and $2000 \Omega$ ) during startup stage; and measurements of slow (scanning rate: $1 \mathrm{mV} \mathrm{s}^{-1}$ ) linear scanning voltammetry on anodes after accumulation in external resistance operation (D).

not produce stable currents at levels close to the catalytic peak currents indicated by the slow LSV measurements.

Figure 2 shows data measured on the activated anode- 400 under operation with a smaller external resistance ( $130 \Omega$, enabling the anode to initially produce high currents close to peak current in insert (b) in Figure 2). The current decreased gradually from $\sim 7.8 \mathrm{~A} \mathrm{~m}^{-2}$ to values even below the stable current generated in the activation stage with an external resistance $400 \Omega$ (shown in insert (a) in Figure 2). The "fragile" characteristics of HER-Anodes had also been observed in the case of polarizing galvanostatically. Generally, HER-Anodes suffered from over-polarization, resulting in anode potential rapidly shift to $>1.2 \mathrm{~V}$ (vs. SHE), within several minutes (data not shown), when subjected to a constant current as high as the peak current indicated in slow LSV curves.

\section{Effect of a series of high ILAC stimulations}

To directly stimulate the development of anode-attached biofilms by high current, ILACs were applied on LER-Anodes and HERAnodes. To run each of ILAC stimulations, slow LSV $\left(1 \mathrm{mV} \mathrm{s}^{-1}\right)$ was first performed on microbial anodes, to determine the peak current (for HER-Anodes) or the limiting current (for LER-Anodes) which were then set as the ILAC-M for the subsequent ILAC operation to stimulate the investigated anode. Each of the ILAC stimulations was performed for 20 hours. Unlike in the case in which bio-anodes were subjected to a constant limiting current, anode-attached biofilms could endure ILACs for prolonged operation without over-polarization (Figure S4). Because the current was controlled at levels as high as the peak/limiting current of the investigated bio-anodes during ILAC application, the anodeattached biofilms were always allowed to grow under a high-current growth environment, unlike the operation by switching MFCs to operation with small external resistance in which high current generated by small external resistance decreased rapidly (Figure 2). Data showed that the series of ILAC stimulations significantly altered the electro activity of the anode-attached biofilms although differences in response to ILACs were observed between LER-Anodes and HER-Anodes. All LER-Anodes (A, B, C, D and E in Figure 3) exhibited similar evolutions of electo catalytic behavior when a series of ILAC stimulations were applied. ILAC stimulations enhanced the catalytic current of LERAnodes from $\sim 3.0 \mathrm{~A} \mathrm{~m}^{-2}$ to $\sim 7.5 \mathrm{~A} \mathrm{~m}^{-2}$ at potentials of $c a .-0.05 \mathrm{~V}$ (vs. SHE), but had no obvious effects in a potential range higher than $0.1 \mathrm{~V}$. Significant change induced by ILAC stimulations for LER-Anodes was that the current-potential profiles exhibited peak currents at a potential of $-0.05 \mathrm{~V}$, similar to that of HER-Anodes. In the case of HER-Anodes, electrochemical behavior (producing peak current in current-potential profiles) did not significantly change, but the electro activity was also found to increase when subjected to ILAC stimulations, especially for anode-1000 and anode-2000 ( $\mathrm{H}$ and I in Figure 3). Under the present stable chemo stat conditions (Figure S2), all bio-anodes with an area of $4 \mathrm{~cm}^{2}(1 \mathrm{~cm} \times 2 \mathrm{~cm} \times 2)$, including LER-Anodes and HER-Anodes, appeared to exhibit significant increase in the electro activity when experiencing the first ILAC stimulations (Figure 3). However, further increase in the electro activity of bio-anodes was generally very limited 


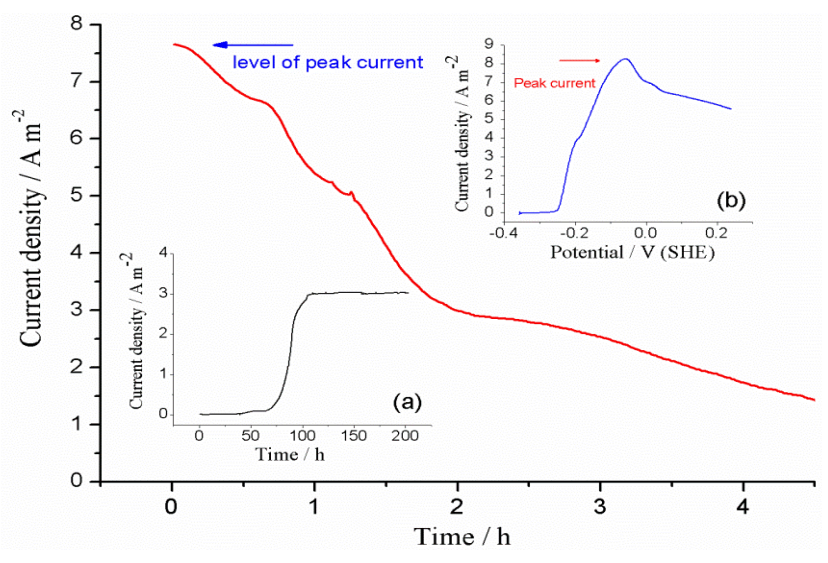

Figure 2: Current variation of the anode previously activated with $400 \Omega$ external resistance operations when switching external resistance to $130 \Omega$. The anode produced a stable current density of ca. $3.0 \mathrm{~A} \mathrm{~m}^{-2}$ (insert (a)) and peak catalytic current of $8.2 \mathrm{~A} \mathrm{~m}^{-2}$ (insert (b)).

when performing high ILAC stimulations beyond the second time (Figure 3).

Figure 4 shows the SEM of anodes of $4 \mathrm{~cm}^{2}$ that were initially activated with different external resistances $(10 \Omega, 50 \Omega, 100 \Omega, 200 \Omega$, $300 \Omega, 400 \Omega, 500 \Omega, 1000 \Omega$ and $2000 \Omega$ ) without ILAC stimulation. Craquelure-like gaps observed in SEM A, B, C, D, E, F, G and H were caused by sample drying. It can be seen that the surface of all these anodes was completely covered by a thick biofilm, except for the anode-2000 (I in Figure 4). SEM showed that the biofilms formed on anode of $4 \mathrm{~cm}^{2}$ contained a large amount of extracellular polymeric substances, exhibiting a structure with compact feature. The compact structure with excess of extracellular polymeric substances might prevent further development of new biofilms, limiting further electro activity increase in biofilms, as shown in Figure 3.

To test the effect of ILAC stimulations on bio-anodes with more blank area for further growth of electro active biofilms, carbon felt anodes with a larger area $\left(9 \mathrm{~cm}^{2}, 3 \mathrm{~cm} \times 1.5 \mathrm{~cm} \times 2\right)$ were first activated to generate a HER-Anode with a thin biofilm by operation with $500 \Omega$ of external resistance for 4 days. When series of ILAC stimulations were applied on HER-Anodes initially with thin biofilms, the electro activity was significantly enhanced, increasing peak/limiting current from $4.8 \pm$ $0.4 \mathrm{~A} \mathrm{~m}^{-2}$ to $10.5 \pm 0.5 \mathrm{~A} \mathrm{~m}^{-2}$ (3 duplicates) (Figure 5C). During series of ILAC stimulations, thin biofilms (Figure 5A) developed to be thick biofilms, completely covering the anode surface (Figure 5B). However, compared with thick biofilms developed in external resistance operation (Figure 4), the thick biofilms (Figure 5B), developed from thin biofilms during series of ILAC stimulations, produced much less extracellular polymeric substances. Less extracellular polymeric substances production enabled the thick biofilms produced by series of ILAC stimulations to have a more porous structure (Figure 5B), In contrast, most bacterial cells in thick biofilms obtained by external resistance operation were found to be embedded in compact extracellular polymeric substances (Figures 4 and S5). Compared with compact structures in thick biofilms, porous structure in thick biofilms would be beneficial to the diffusion of nutrients and metabolic wastes [31,32], consequently reducing the effect of diffusion on high current. Remarkably, the ILAC-induced thick porous biofilms, developed from thin biofilms, showed considerable robustness. When bio-anodes with the ILAC-induced thick porous biofilms were transferred to operation with a continuous current of $9.5 \mathrm{~A} \mathrm{~m}^{-2}$, close to their limiting currents, stable power outputs of $4.35 \pm 0.27 \mathrm{~W} \mathrm{~m}^{-2}$ in the present MFC setup with chemostat conditions (i.e., $\mathrm{pH}$ and concentration of organic substrate) were produced without obvious decay in performance during prolonged operation (Figure S6).

\section{Current adaptation for robust electro-active biofilms}

Bio-anodes with electro-active biofilms are key components in bio-electrochemical systems. In microbial anodes, the current is a measure of the rate of the extracellular electron transfer by electroactive microorganisms. During potential-adaptive growth of electro active biofilms, electric current, as a dependent parameter, is governed partially by the structure, diffusion, biomass and physiological activity of the electro-active biofilms [13,29,33]. For instance, current would be limited in biofilms with compact structures, slow nutrient diffusion and ineffective microorganisms. However, the present study indicated that the current could conversely influence the development of electroactive biofilms, and offer anode-attached biofilms opportunities to develop high current-adaptive capability when it was controlled as an active factor to stimulate the growth of biofilms, presumably through establishing an optimized biofilm structures, and accelerating the growth rate of microbial species with high effective electro-activity, at least in the sensitive startup period. Furthermore, to flow current was also shown to be crucial for maintaining the performance of the anodic biofilms that had evolved to have a high electro catalytic capacity, as illustrated in Figure 6. When the bio-anodes with high performance were disconnected from the circuit, no current flowed through the anode-attached biofilms. As a result, the electro activity decreased significantly (curves 2, 3 and 4 in Figure 6). Performance restoration of electro active anode-attached biofilms required current flowing through biofilms again (curve 5 in Figure 6).

In the present study, instead of potential, high currents were used to directly stimulate the development of electro-active biofilms by intermittent connection/disconnection operation. Recently, some strategies based on intermittent connection/disconnection operation were used to modulate the anode potential duration [24], external resistance loading [25,26] and MFC voltage [27]. However the current was still a dependent parameter in those reported strategies. In contrast, in the ILAC method presented here, the current functioned as an active electrochemical parameter, rather than as a dependent parameter, to directly stimulate the development of microbial anodes. ILAC stimulations were found to have positive effects on the current adaptive capacity of anodic biofilms in the present study, resulting in high electro activity and robustness. The present study suggested an alternative method for establishing anodic biofilms with high performance in future bio electrochemical system development.

\section{Conclusions}

Electro-active biofilms formed on anodes in bio electrochemical systems with different external resistance exhibited different electrochemical behavior. Microbial anodes that were activated with external resistance operation were observed to lack the ability to endure high current, but could endure the impact of ILACs without over-polarization. The application of an ILAC provided a method to directly stimulate the development of anode-attached biofilms by high currents, and has been shown to be effective for enhancing the electro-activity of anodic biofilms, especially for anodes with pre-formed thin biofilms. Robustness of anode-attached biofilms could be obtained by high current stimulations. The study showed that current, as a controlling parameter, is a crucial factor influencing the performance of electro-active biofilms, presumably through optimizing the structures in anode-attached biofilms for current production. 
Citation: Zhang E, Yu Q, Zhang Y, Scott K, Diao G (2017) The Effect of Intermittent Limiting Anodic Current Stimulation on the Electro Activity of Anodic Biofilms. J Adv Chem Eng 7: 174. doi: 10.4172/2090-4568.1000174
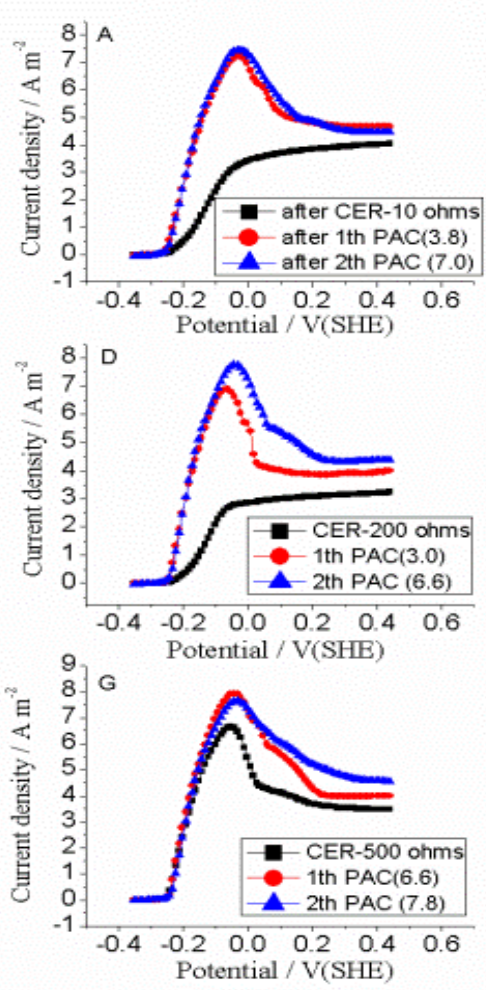
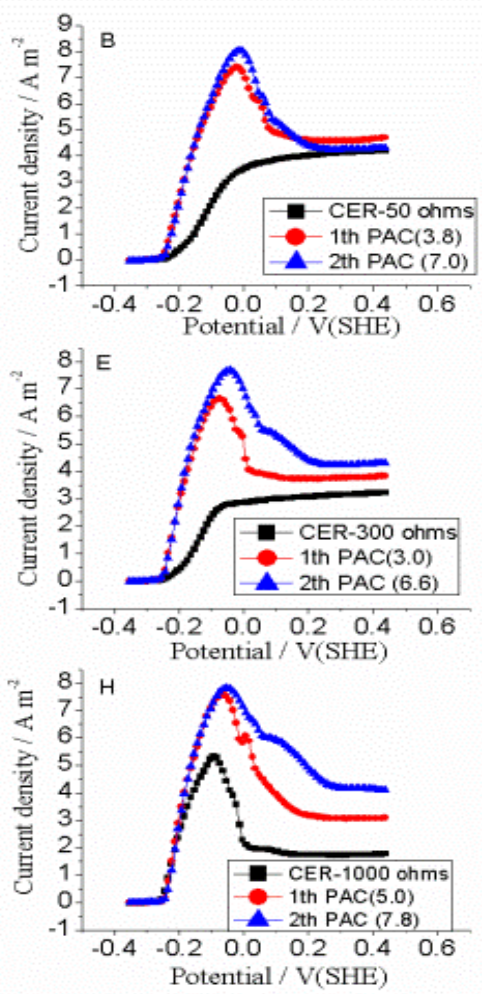
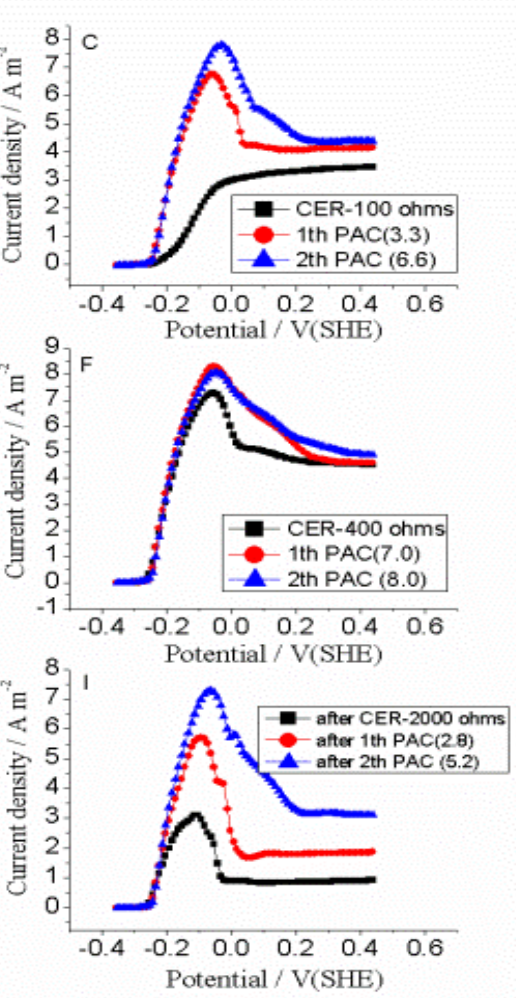

Figure 3: Evolvements of electro-activities on anodes $(1 \mathrm{~cm} \times 2 \mathrm{~cm} \times 2)$ with thick biofilms activated with different external resistances $(A: 10 \Omega$, B: $50 \Omega$, C: $100 \Omega$, D: $200 \Omega$, E: $300 \Omega, F: 400 \Omega$, G: $500 \Omega, H: 1000 \Omega$ and I: $2000 \Omega$ ) when experiencing series of high ILAC stimulations. The electro-activities were characterized by slow LSV (scan rate: $1 \mathrm{mV} \mathrm{s}^{-1}$ ). Taking figure A as an example to explain the legends: 'CER-10 ohms' refers to the measurement on the anode after pre-activation with constant external resistance of $10 \Omega$; '1th ILAC (3.8)' refers to the measurement on the same anode experiencing the first ILAC stimulation with the current 3.8 $\mathrm{A} \mathrm{m}^{-2}$; '2th ILAC (7.0) refers to the measurement on the same anode experiencing the second ILAC stimulation with the current $7.0 \mathrm{~A} \mathrm{~m}^{-2}$.
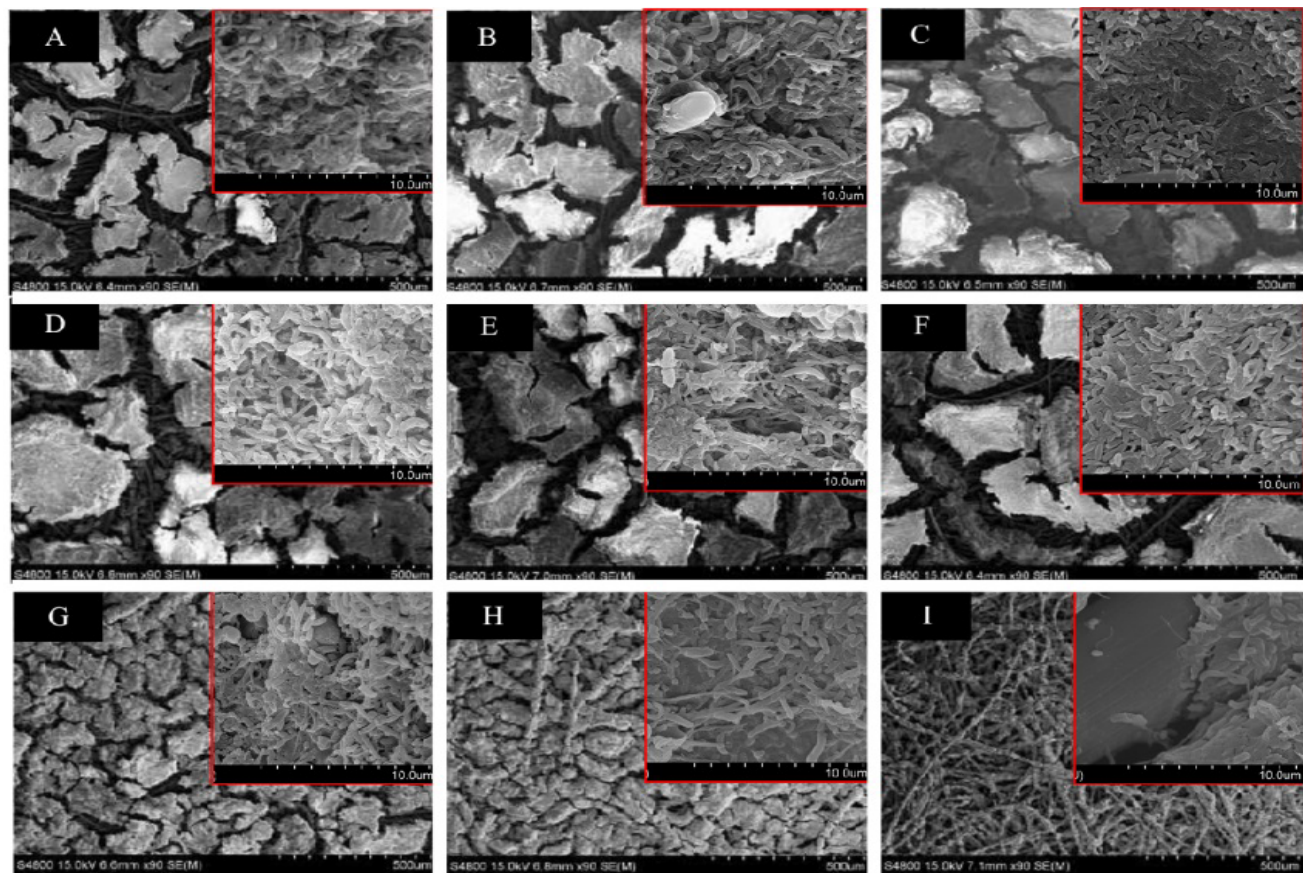

Figure 4: SEM of biofilms formed on anodes pre-activated with different external resistance (A: $10 \Omega, B: 50 \Omega, C: 100 \Omega$, D: $200 \Omega$, E: $300 \Omega$, F: $400 \Omega$, G: $500 \Omega, \mathrm{H}: 1000 \Omega$ and I: $2000 \Omega$ ). 
Citation: Zhang E, Yu Q, Zhang Y, Scott K, Diao G (2017) The Effect of Intermittent Limiting Anodic Current Stimulation on the Electro Activity of Anodic Biofilms. J Adv Chem Eng 7: 174. doi: 10.4172/2090-4568.1000174

Page 6 of 7
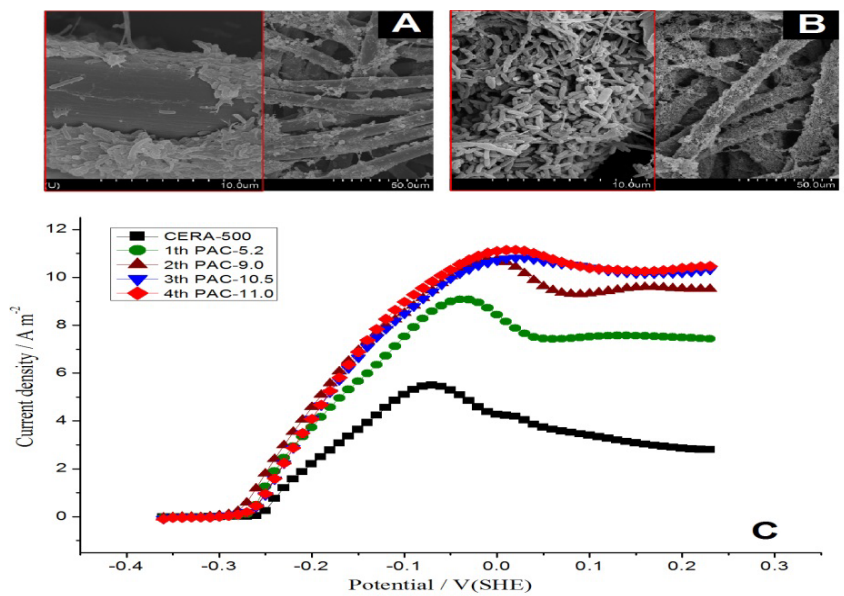

Figure 5: Thin anodic biofilms with more blank area before ILAC stimulations (A), thick porous anodic biofilms developed from thin biofilms experiencing four ILAC stimulations (B) and slow LSV measurements during series of ILAC application. To understand the legends in $\mathrm{C}$, please turn to Figure 3.

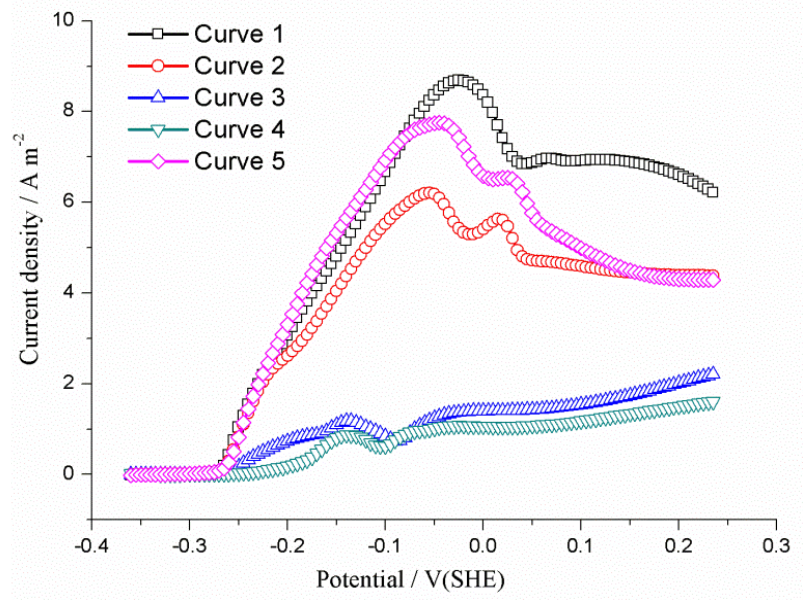

Figure 6: Slow linear sweep voltammograms measured on the same bioanode at different working states (potential scan rate: $1 \mathrm{mV} \mathrm{s}^{-1}$ ). Curve 1 represents the result for the anodic biofilm with good performance. Curve 2, 3, 4 represent the results for the anodic biofilm experiencing disuse (zero current) for 24, 72 and 96 hours, respectively. Curve 5 represents the result for the anodic biofilm experiencing 10 hours re-connection in circuit with fixed external resistance $(500 \Omega)$ after the measurement of curve 4 .

\section{Acknowledgments}

This work was financially supported by the National Natural Science Foundation of China (Grant No. 21173184) and a Project Funded by the Priority Academic Program Development of Jiangsu Higher Education Institutions.

\section{References}

1. Logan BE (2009) Exoelectrogenic bacteria that power microbial fuel cells. Nat Rev Microbiol 7: 375 .

2. Halan B, Buehler K, Schmid A (2012) Biofilms as living catalysts in continuous chemical syntheses. Trends Biotechnol 30: 453.

3. Logan BE, Rabaey K (2012) Conversion of Wastes into Bioelectricity and Chemicals by Using Microbial Electrochemical Technologies. Science 337: 686-690.

4. Bajracharya S, Sharma M, Mohanakrishna G, Dominguez Benneton XD, Strik DPBTB, et al. (2016) An overview on emerging bio electrochemical systems
(BESs): Technology for sustainable electricity, waste remediation, resource recovery, chemical production and beyond. Renew Energ 98: 153.

5. Feng C, Huang L, Yu H, Yi X, Wei C (2015) Simultaneous phenol removal, nitrification and denitrification using microbial fuel cell technology. Water Res 76: 160

6. Ismail ZZ, Habeeb AA (2017) Experimental and modeling study of simultaneous power generation and pharmaceutical wastewater treatment in microbial fuel cell based on mobilized biofilm bearers. Renew Energ 101: 1256.

7. Hasany M, Mardanpour MM, Yaghmaei S (2016) Biocatalysts in microbial electrolysis cells: A review. Int J Hydrogen Energy 41: 1477.

8. LaBarge N, Yilmazel YD, Hong PY, Logan BE (2017) Effect of pre-acclimation of granular activated carbon on microbial electrolysis cell start up and performance. Bio electrochemistry 113: 20.

9. Rago L, Baeza JA, Guisasola A (2016) Increased performance of hydrogen production in microbial electrolysis cells under alkaline conditions. Bioelectrochemistry 109: 57.

10. Di Lorenzo M, Thomson AR, Schneider K, Cameron PJ, leropoulos I (2014) A small-scale air-cathode microbial fuel cell for on-line monitoring of water quality. Biosens Bioelectron 62: 182

11. Cheng L, Quek SB, Cord-Ruwisch R (2014) Hexacyanoferrate-adapted biofilm enables the development of a microbial fuel cell biosensor to detect trace levels of assimilable organic carbon $(A O C)$ in oxygenated seawater. Biotechnol Bioeng 111: 2412

12. Rasmussen M, Minteer SD (2015) Long-term arsenic monitoring with an Enterobacter cloacae microbial fuel cell. Bioelectrochemistry 106: 207.

13. Borole AP, Reguera G, Ringeisen B, Wang ZW, Feng Y, et al. (2011) Electroactive biofilms: Current status and future research needs. Energ Environ Sci 4: 4813-4834.

14. Yun $H$, Kong D, Liang B, Cui M, Li Z, et al. (2016) Response of anodic bacteria community to the polarity inversion for chloramphenicol reduction. Bioresour Technol 221: 666 .

15. Ullery ML, Logan BE (2015) Anode acclimation methods and their impact on microbial electrolysis cells treating fermentation effluent. Int J Hydrogen Energy 40: 6782 .

16. Bridier A, Quemener ED, Bureau C, Champigneux P, Renvoise L, et al. (2015) Successive bioanode regenerations to maintain efficient current production from biowaste. Bio electrochemistry 106: 133.

17. Cercado B, Byrne N, Bertrand M, Pocaznoi D, Rimboud M, et al. (2013) Garden compost inoculum leads to microbial bioanodes with potential-independent characteristics. Bioresour Technol 134: 276.

18. Zhu X, Yates MD, Hatzell MC, Rao H, Ananda PE (2014) Microbial Community Composition Is Unaffected by Anode Potential. Environ Sci Technol 48: 1352-1358.

19. Villano M, Ralo C, Zeppilli M, Aulenta F, Majone M (2016) Influence of the set anode potential on the performance and internal energy losses of a methaneproducing microbial electrolysis cell. Bio electrochemistry 107: 1.

20. Rousseau R, Santaella C, Bonnafous A, Achouak W, Godon JJ (2016) Halotolerant bioanodes: The applied potential modulates the electrochemical characteristics, the biofilm structure and the ratio of the two dominant genera. Bio electrochemistry 112: 24

21. Zhu X, Tokash JC, Hong Y, Logan BE (2013) Controlling the occurrence of power overshoot by adapting microbial fuel cells to high anode potentials. Bio electrochemistry 90: 30 .

22. Winfield J, leropoulos I, Greenman J, Dennis J (2011) The overshoot phenomenon as a function of internal resistance in microbial fuel cells. Bio electrochemistry 81: 22.

23. Nien PC, Lee CY, Ho KC, Adav SS, Liu L, et al. (2011) Power overshoot in twochambered microbial fuel cell (MFC). Bioresour Technol 102: 4742.

24. Gardel EJ, Nielsen ME, Grisdela PT, Girguis PR (2012) Duty Cycling Influences Current Generation in Multi-Anode Environmental Microbial Fuel Cells. Environ Sci Techno 46: 5222-5229.

25. Grondin F, Perrier M, Tartakovsky B (2012) Microbial fuel cell operation with intermittent connection of the electrical load. J Power Sources 208: 18.

26. Coronado J, Perrier M, Tartakovsky B (2013) Pulse-width modulated externa resistance increases the microbial fuel cell power output. Bioresour Techno $147: 65$ 
Citation: Zhang E, Yu Q, Zhang Y, Scott K, Diao G (2017) The Effect of Intermittent Limiting Anodic Current Stimulation on the Electro Activity of Anodic Biofilms. J Adv Chem Eng 7: 174. doi: 10.4172/2090-4568.1000174

27. Nandy A, Kumar V, Kundu PP (2016) Effect of electric impulse for improved energy generation in mediatorless dual chamber microbial fuel cell through electroevolution of Escherichia coli. Biosens Bioelectron 79: 796.

28. Zhang L, Zhu X, Li J, Liao Q, Ye D (2011) Biofilm formation and electricity generation of a microbial fuel cell started up under different external resistances. J Power Sources 196: 6029.

29. Li N, Kakarla R, Min B (2016) Effect of influential factors on microbial growth and the correlation between current generation and biomass in an air cathode microbial fuel cell. Int J Hydrogen Energy 41: 20606.

30. Hong Y, Call DF, Werner CM, Logan BE (2011) Adaptation to high current using low external resistances eliminates power overshoot in microbial fuel cells. Biosens Bioelectron 28: 71
31. Karthikeyan R, Wang B, Xuan J, Wong JWC, Lee PKH (2015) Interfacial electron transfer and bioelectrocatalysis of carbonized plant material as effective anode of microbial fuel cell. Electrochim Acta 157: 314.

32. Sharma M, Bajracharya S, Gildemyn S, Patil SA, Alvarez-Gallego Y, et al. (2014) A critical revisit of the key parameters used to describe microbia electrochemical systems. Electrochim Acta 140: 191.

33. Korth B, Rosa LFM, Harnisch F, Picioreanu C (2015) A framework for modeling electroactive microbial biofilms performing direct electron transfer. Bioelectrochemistry 106: 194. 